\section{(A) Check for updates}

Cite this: Analyst, 2021, 146, 4895

\title{
Insight into metastatic oral cancer tissue from novel analyses using FTIR spectroscopy and aperture IR-SNOM
}

\author{
Barnaby G. Ellis, ${ }^{a}$ Conor A. Whitley, ${ }^{a}$ Safaa Al Jedani, ${ }^{a}$ Caroline I. Smith, (D) ${ }^{a}$ \\ Philip J. Gunning, (D) baul Harrison, ${ }^{\mathrm{b}}$ Paul Unsworth, ${ }^{a}$ Peter Gardner, (D) c \\ Richard J. Shaw, ${ }^{\text {,d }}$ Steve D. Barrett, (D) a Asterios Triantafyllou, (D) e Janet M. Risk (D) ${ }^{\text {b }}$ \\ and Peter Weightman (D) *a
}

\begin{abstract}
A novel machine learning algorithm is shown to accurately discriminate between oral squamous cell carcinoma (OSCC) nodal metastases and surrounding lymphoid tissue on the basis of a single metric, the ratio of Fourier transform infrared (FTIR) absorption intensities at $1252 \mathrm{~cm}^{-1}$ and $1285 \mathrm{~cm}^{-1}$. The metric yields discriminating sensitivities, specificities and precision of $98.8 \pm 0.1 \%, 99.89 \pm 0.01 \%$ and $99.78 \pm$ $0.02 \%$ respectively, and an area under receiver operator characteristic (AUC) of $0.9935 \pm 0.0006$. The delineation of the OSCC and lymphoid tissue revealed by the image formed from the metric is in better agreement with an immunohistochemistry $(\mathrm{IHC})$ stained image than are either of the FTIR images obtained at the individual wavenumbers. Scanning near-field optical microscopy (SNOM) images of the tissue obtained at a number of key wavenumbers, with high spatial resolution, show variations in the chemical structure of the tissue with a feature size down to $\sim 4 \mu \mathrm{m}$. The image formed from the ratio of the SNOM images obtained at $1252 \mathrm{~cm}^{-1}$ and $1285 \mathrm{~cm}^{-1}$ shows more contrast than the SNOM images obtained at these or a number of other individual wavenumbers. The discrimination between the two tissue types is dominated by the contribution from the $1252 \mathrm{~cm}^{-1}$ signal, which is representative of nucleic acids, and this shows the OSCC tissue to be accompanied by two wide arcs of tissue which are particularly low in nucleic acids. Haematoxylin and eosin (H\&E) staining shows the tumour core in this specimen to be $\sim 40 \mu \mathrm{m}$ wide and the SNOM topography shows that the core centre is raised by $\sim 1 \mu \mathrm{m}$ compared to the surrounding tissue. Line profiles of the SNOM signal intensity taken through the highly keratinised core show that the increase in height correlates with an increase in the protein signal. SNOM line profiles show that the nucleic acids signal decreases at the centre of the tumour core between two peaks of higher intensity. All these nucleic acid features are $\sim 25 \mu \mathrm{m}$ wide, roughly the width of two cancer cells.
\end{abstract}

Received 24th May 2021, Accepted 1st July 2021

DOI: 10.1039/d1an00922b rsc.li/analyst

\section{Introduction}

There is considerable interest in the detection of cancer by applying machine learning algorithms to the analysis of the extensive datasets obtained by the application of infrared (IR)

\footnotetext{
${ }^{a}$ Department of Physics, University of Liverpool, L69 7ZE, UK.

E-mail: peterw@liverpool.ac.uk

${ }^{b}$ Department of Molecular and Clinical Cancer Medicine, Institute of Systems, Molecular and Integrative Biology, University of Liverpool, L3 9TA, UK

${ }^{c}$ Manchester Institute of Biotechnology, 131 Princess Street, University of Manchester, Manchester, M1 TDN, UK

${ }^{d}$ Regional Maxillofacial Unit, Aintree University Hospital, Liverpool, L9 7AL, UK ${ }^{e}$ Department of Pathology, Liverpool Clinical Laboratories, University of Liverpool, Liverpool, L69 3GA, UK
}

imaging spectroscopies to fixed human tissue. $^{1-9}$ Baker et al. ${ }^{10,11}$ demonstrated considerable improvement in sensitivity and specificity in the Gleason grading of prostate cancer when applying principal component discriminant function analysis (PC-DFA) to a Fourier transform infrared (FTIR) imaging dataset. Similarly, the application of convolutional neural networks to a combination of results obtained from FTIR spectral imaging and associated spatial information obtained from tissue microarrays was able to identify six major cellular and acellular constituents associated with breast cancer. ${ }^{3}$

There have been several reviews of advances in the instrumentation and application of the FTIR technique to cancer $^{12-15}$ and the application of techniques for obtaining chemical information from FTIR. ${ }^{3,6-9}$ We recently applied a novel machine learning multivariate metrics analysis (MA) 
technique to the analysis of FTIR images obtained from four cell lines associated with oesophageal cancer ${ }^{16}$ and compared its performance with the well-established random forest (RF) method. The MA was found to achieve greater accuracy in discriminating between the cell types in a shorter time than the RF method. In particular the MA was able to discriminate with accuracies in the range of $81 \%$ to $97 \%$ between OE19 and OE21 cell lines, associated respectively with adenocarcinoma and squamous carcinoma, and more importantly between cancer associated myofibroblasts (CAM) and adjacent tissue myofibroblasts (ATM) obtained from the same patient. In addition to discriminating between these cell lines, the MA yielded a number of key spectral biomarkers that had not been identified in previous FTIR studies of oesophageal cancer.

FTIR and Raman imaging has previously been applied to the discrimination of oral cancer from histologically normal or benign tissue in a number of studies. ${ }^{17}$ For example, Pallua et al. $^{18}$ used principal component analysis (PCA) and cluster analysis to produce pseudo-colour images of oral squamous cell carcinoma (OSCC) tissue microarrays and showed correspondence between FTIR and routine histology, suggesting that tissue types are separable by their IR spectra when appropriate methods are used to analyse the dataset. Lloyd et al. ${ }^{2}$ developed a multivariate analysis technique that combined PCA followed by linear discriminant analysis (LDA) to results obtained by Raman spectroscopy. This was able to discriminate between lymph nodes with benign pathology from those harbouring lymphoma or metastases of head and neck cancer with sensitivities and specificities of $81 \%$ and $89 \%$ respectively. Another study ${ }^{19}$ used a framework of feature selection and classification algorithms to identify spectral features which distinguished normal mucosa, pre-cancerous tissue and cancer of the oral cavity. Particular wavenumbers, previously correlated with chemical moieties such as glycogen and proteins, were discriminatory which suggests that relevant information comparable to that previously obtained via other methodologies is achievable from such data. A comprehensive review of Raman and FTIR studies of oral cancers has recently been published by Byrne et al. ${ }^{17}$

The present investigation examines the value of the MA technique in discriminating between lymph nodal metastasis of oral cancer and indigenous lymphoid tissue. High spatial resolution measurements using an aperture scanning nearfield optical microscope (SNOM) provide additional insight into the chemical biology of the metastatic tissue.

\section{Experimental}

\section{Preparation of samples for analysis}

Archival formalin-fixed, paraffin-embedded (FFPE) tissue from cervical lymph node metastases were obtained from a single patient with OSCC following informed consent. All experiments were performed in accordance with University of Liverpool guidelines; with the sponsorship of their Joint Research Office and with ethical approval from the Northwest
Liverpool Central Research Ethics Committee (REC number EC 47.01).

Regions of interest (ROIs) $(n=2)$ containing both metastatic OSCC and surrounding lymphoid tissue were identified by light microscopy on sections routinely prepared and stained with haematoxylin and eosin (H\&E). Cores of $1 \mathrm{~mm}$ diameter corresponding to the ROIs were then obtained from the FFPE blocks using a Beecher MTA-1 tissue microarrayer for constructing a tissue microarray block. Serial, $5 \mu \mathrm{m}$ thick, sections were cut from the tissue microarray block and floated onto charged glass slides for histopathology and immunohistochemistry (IHC) and onto calcium fluoride $\left(\mathrm{CaF}_{2}\right)$ disks for FTIR imaging. While sections for IHC were eventually subjected to deparaffinisation, sections for FTIR remained in paraffin wax to minimise further changes in hydration and structure of the samples. Six serial sections were utilised and comprised two sections for FTIR imaging sandwiched between two sections stained with H\&E and two with IHC for pan-cytokeratins using the AE1AE3 antibody (Agilent DAKO, Stockport, $\mathrm{UK}$ ) and a Bond $\mathrm{RX}^{\mathrm{TM}}$ autostainer (Leica Biosystems, Milton Keynes, UK). The H\&E and IHC stained sections were scanned using an Aperio CS2scanner (Leica Biosystems) to facilitate coregistration with IR images.

\section{FTIR experiments}

Mid-IR spectroscopic images were acquired from each ROI using an Agilent Cary 620 FTIR microscope coupled to an Agilent Cary 670 FTIR spectrometer (Agilent, Stockport, UK) as described previously. ${ }^{16,20}$ Poor quality spectra, defined as having an amide I absorbance (peak centre $1650 \mathrm{~cm}^{-1}$ ) less than 0.1 or greater than 2 , were removed from the dataset. This range was chosen so that outlier spectra arising from suboptimal sample thickness would be discarded whilst retaining the vast majority of data. The spectra were then truncated to the fingerprint region ( $\left.900 \mathrm{~cm}^{-1}-1800 \mathrm{~cm}^{-1}\right)$ and the region dominated by paraffin contributions $\left(1350 \mathrm{~cm}^{-1}-1500 \mathrm{~cm}^{-1}\right)$ was omitted from the analysis. Each spectrum in the truncated dataset was then subject to a rubber-band baseline correction, ${ }^{21}$ followed by vector normalisation. Corrections for Mie scattering are unnecessary for FFPE tissue due to the refractive index matching between the tissue and paraffin, thus significantly reducing scattering artefacts. ${ }^{13}$

The histopathological and FTIR images were cross-referenced and spectra from the ROIs were identified and labelled as OSCC or lymphoid tissue as appropriate. Labelled FTIR data were used to train a discriminatory model using the MA technique. ${ }^{16} \mathrm{MA}$ is a supervised learning technique which generates an ensemble of bivariate classifiers based on the ratio of absorbances for all pairings of wavenumber features in the data. Through an iterative approach, it seeks to determine the ratios which provide the best classification accuracy, incorporating the top ranking metrics into a dynamic hard-voting ensemble classifier. The main advantage of this approach is that it is a more direct measure of feature importance - a cumulative importance histogram is obtained, rather than a multivariate weight vector that results from classifiers such as 
logistic regression and linear discriminant analysis. An equal number of spectra were randomly sampled from each image so as to mitigate the risk of overfitting to image-specific features. The MA model was trained using a three-fold cross validation regime, whereby the data is divided into three partitions, selecting two for training and holding out the third for testing. This process is repeated three times so that all data appears in both the training and testing sets.

\section{SNOM experiments}

Experiments were also performed using an aperture SNOM described previously. ${ }^{20,22-25}$ The infrared source was a quantum cascade laser (QCL) instrument (Daylight Solutions, San Diego, USA), equipped with three modules enabling an effective spectral range of $1965 \mathrm{~cm}^{-1}-1145 \mathrm{~cm}^{-1}$ and pulsing at a rate of $80 \mathrm{kHz}$ with pulse widths of 200-500 ns. The $x-y$ piezo-stage was configured to scan a region of $300 \times 300 \mu \mathrm{m}$ with a step of $2 \mu \mathrm{m}$. The SNOM imaging tip was a specially prepared IR-transmitting chalcogenide fibre (CorActive, Quebec, Canada) of core diameter $100 \mu \mathrm{m}$, sharpened by etching, to create a small aperture through which the SNOM images were collected $^{26}$ concurrently with shear-force topography. The images were corrected for non-linearity of the piezo stage, and other common processing techniques such as streak removal and line-levelling were applied. The images were co-registered using cross correlations and then a Gaussian smoothing of 2 pixels $(4 \mu \mathrm{m})$, full-width half-maximum (FWHM) was applied.

\section{Results}

\section{Discrimination of OSCC metastases from lymph node tissue}

The MA algorithm produces a ranked list of metrics, an ensemble of which produces the optimum discrimination. ${ }^{16}$ The trained MA model was able to discriminate between metastatic OSCC and the surrounding lymphoid tissue with a high sensitivity and specificity by utilising only the highest-ranking metric, specifically the ratio of intensities at $1252 \mathrm{~cm}^{-1}$ and $1285 \mathrm{~cm}^{-1}$ (Table 1). The success of this metric is shown in Fig. 1(a) in which the histograms of the ratio of the intensities of the discriminating wavenumbers obtained at each pixel in the areas of the FTIR images identified with each tissue type in the images used to train the algorithm are plotted. This shows that the test spectra conform very well with the decision boundaries formed by this metric, explaining the high AUC.

Table 1 Measures of discrimination between metastatic OSCC and lymphoid nodal tissue for the highest-ranking metric. The mean and standard deviation are taken from across three cross validation partitions

\begin{tabular}{lc}
\hline Highest ranked metric & $1252 \mathrm{~cm}^{-1} / 1285 \mathrm{~cm}^{-1}$ \\
\hline Sensitivity & $98.8 \pm 0.1 \%$ \\
Specificity & $99.89 \pm 0.01 \%$ \\
Precision & $99.78 \pm 0.02 \%$ \\
Area under curve (AUC) $^{a}$ & $0.9935 \pm 0.0006$ \\
${ }^{a}$ Area under the receiver operating characteristic (ROC) curve.
\end{tabular}
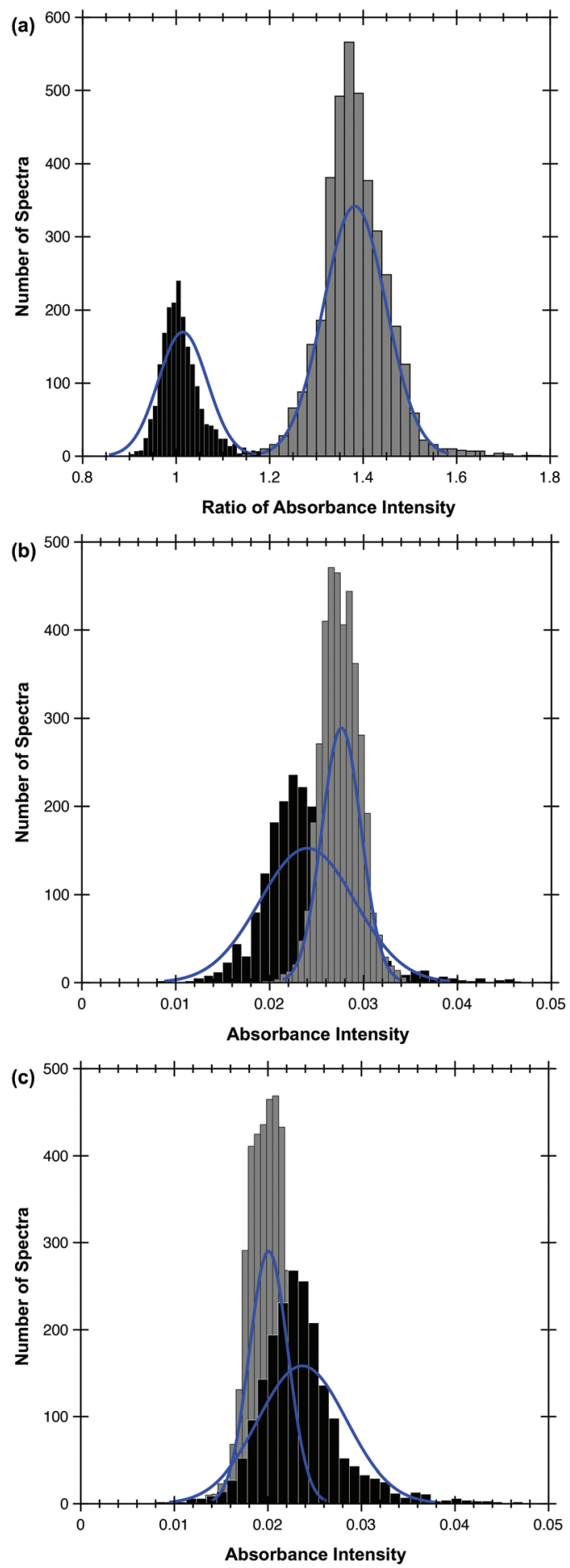

Fig. 1 (a) The normal distributions (blue lines) fitted to the training data (not shown) for OSCC and lymphoid tissue. Histograms of OSCC (black) and lymphoid tissue (grey) testing spectra are also shown. Histograms for (b) $1252 \mathrm{~cm}^{-1}$ and (c) $1285 \mathrm{~cm}^{-1}$ show more overlap and hence explain the poorer values for sensitivity and specificity quoted in the text.

These two wavenumbers and those contained in the next four metrics in rank order, 1254/1285, 1250/1289, 1252/1287 and $1252 / 1289$, draw attention to a very narrow region of the 


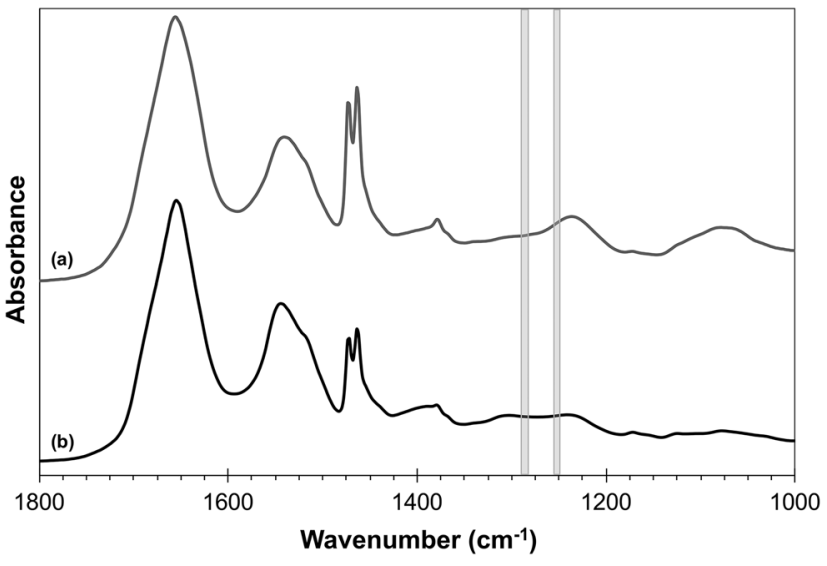

Fig. 2 Average FTIR profiles for (a) lymphoid tissue (grey) spectra and (b) OSCC (black). The shaded grey rectangles show the regions of $1250-1254 \mathrm{~cm}^{-1}$ and $1285-1289 \mathrm{~cm}^{-1}$.

FTIR spectrum, wherein the average spectra of different types of tissue show differences (Fig. 2).

This highest-ranking metric discriminates between OSCC and lymphoid tissue better than the individual wavenumbers (Fig. 3). If the absorbance at $1252 \mathrm{~cm}^{-1}$ and $1285 \mathrm{~cm}^{-1}$ were used individually as discriminatory features, the performance of the model would drop significantly. The sensitivity and specificity obtained by using $1252 \mathrm{~cm}^{-1}$ alone would be $89.3 \%$ and $73.3 \%$ respectively; the corresponding results acquired using $1285 \mathrm{~cm}^{-1}$ alone would be $90.4 \%$ and $54.4 \%$ respectively. This is illustrated by the normal distributions shown in Fig. 1(b) and (c). Thus, although a correspondence between tumour cells stained by IHC [Fig. 3(a)] and the low absorbance at $1252 \mathrm{~cm}^{-1}$ [Fig. 3(c)] is observed, a greater correlation is seen between the IHC and the ratio of $1252 \mathrm{~cm}^{-1} / 1285 \mathrm{~cm}^{-1}$ [Fig. 3(d)]. However, topographically different areas of the metastasis (e.g. periphery versus the more heavily keratinised centre as appreciated on H\&E sections) are not discriminated by the metric (Fig. 3).

\section{SNOM analysis of OSCC nodal metastases}

To further investigate the biological changes that underly the discriminatory metric, a second core from a different region in the same lymph node metastasis specimen shown in Fig. 3 was dewaxed using the protocol described recently ${ }^{20}$ and topography and SNOM images were obtained of a small region of this tissue that contained the OSCC-lymphoid tissue interface. The results obtained in these experiments are shown in Fig. 4. Fig. 4(a) and (b) show the H\&E and IHC stained images of this region of the core, respectively, and Fig. 4(c) shows the topography obtained during the collection of SNOM images. SNOM images were collected at a number of wavenumbers that have been shown to be important in discriminating oesophageal cancer cells ${ }^{24,25}$ and in the development of a dewaxing protocol for SNOM experiments: ${ }^{20} 1751 \mathrm{~cm}^{-1}, 1650 \mathrm{~cm}^{-1}, 1369 \mathrm{~cm}^{-1}$ (shown in Fig. 4(d), (e) and (f), respectively). The SNOM images obtained at the discriminating wavenumbers $1285 \mathrm{~cm}^{-1}$ and $1252 \mathrm{~cm}^{-1}$, defined above, are shown in Fig. $4(\mathrm{~g})$ and $(\mathrm{h})$, respectively, and the ratio of the intensity of these two images is shown in Fig. 4(i). The images indicate presence of tumour mass in the bottom right corner of the image, while the heterogeneity of the images indicates that additional, higher resolution, differences might also be identified in the tissue by this method.

In order to bring out in more detail the information captured in the images obtained with high spatial resolution using the SNOM (Fig. 4), the smaller region of the tumour in the bottom right-hand corner of the H\&E image [Fig. 4(a)] was used to create line profiles of the topography and the SNOM intensities at each wavenumber. Each profile was obtained along a 1-pixel-wide line close to the centre of the OSCC nodal metastasis (Fig. 5). The noise levels in the SNOM images (and hence the profiles) were quantified by comparing raw images with de-noised images, and the noise-to-signal ratios were found to be $<5 \%$ for all wavenumbers. Line profiles taken within 8 microns of those shown in Fig. 5 show only very small differences from those shown in the figure. The topography [Fig. 5(a)] of the centre of the tumour can be seen to be higher than the surrounding tissue. This increase in height correlates with an increase in the protein signal [Fig. 5(c)] in this region of the image. The line profiles obtained at other wavenumbers show more marked variations in intensity across smaller distances, indicating that there are many subtle changes in the chemistry of the metastasis.

The SNOM images were taken in IR transmission mode and so the SNOM intensity profiles in Fig. 5 have been inverted to present a more intuitive interpretation - peaks (valleys) in the profiles correspond to more (less) absorption. The profiles are presented on vertical scales that have been corrected for image acquisition parameters such as detector sensitivity. Comparison between profiles at different wavenumbers should not be taken as providing values for relative molecular concentrations, as the SNOM fibre transmission varies with wavenumber and each molecular vibration has a different transition dipole strength.

\section{Discussion}

The MA algorithm applied to FTIR data is able to discriminate between OSCC nodal metastases and surrounding lymphoid tissue on the basis of a single metric, the ratio of intensities at $1252 \mathrm{~cm}^{-1}$ and $1285 \mathrm{~cm}^{-1}$ (Table 1). Although there is a correspondence between the FTIR image obtained at $1252 \mathrm{~cm}^{-1}$ [Fig. 3(c)] and IHC [Fig. 3(a)], the image formed from the ratio of the intensities of the images obtained at $1252 \mathrm{~cm}^{-1}$ and $1285 \mathrm{~cm}^{-1}$ [Fig. 3(d)] seems in better agreement with the IHC than are either of the images obtained at the individual wavenumbers. This is to be expected given the high specificity, sensitivity and precision attributed to this metric during the MA of the FTIR spectra [Table 1, Fig. 1(a)]. It is apparent that, with sufficient spatial resolution and control of the signal-to-noise 

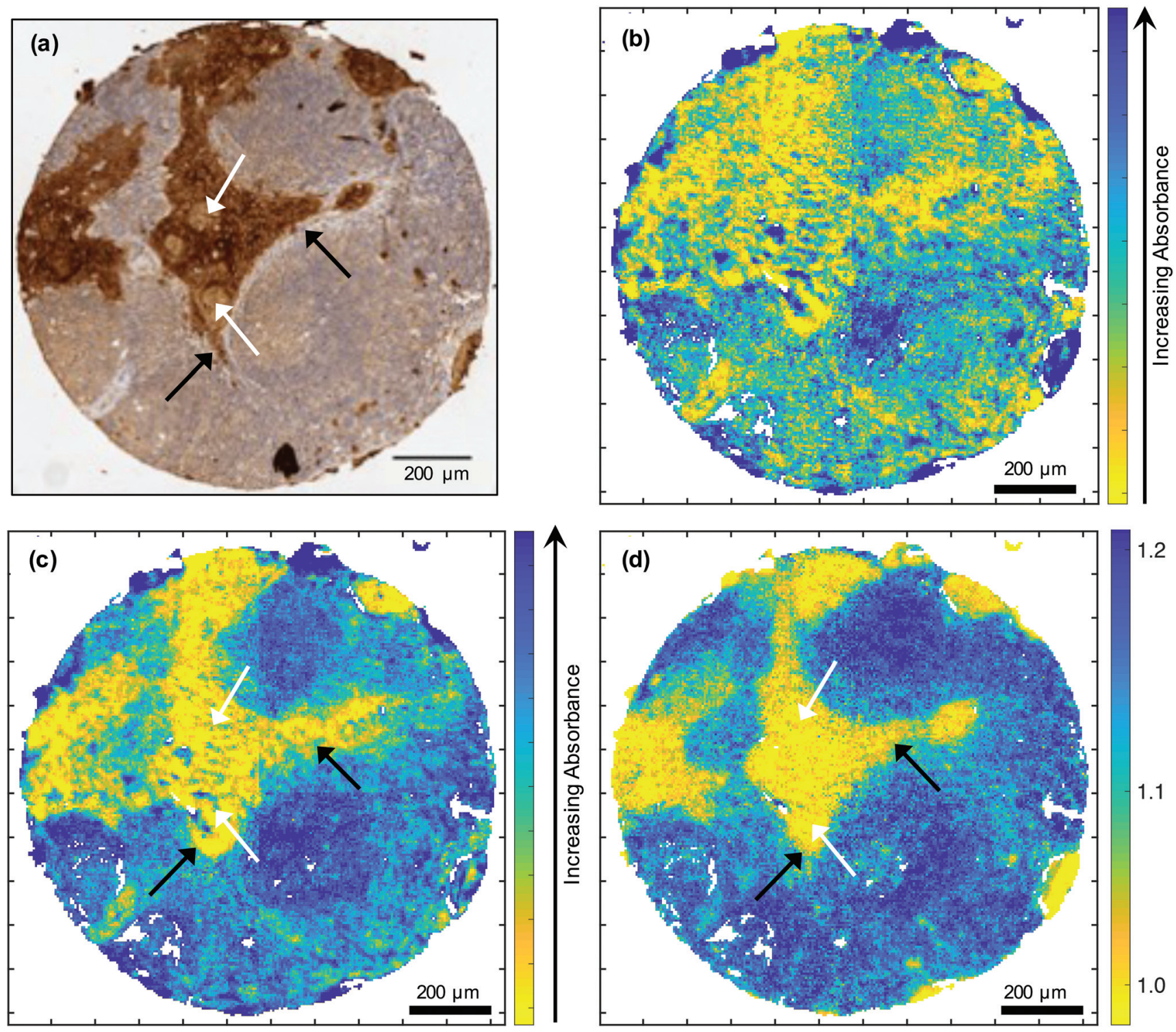

Fig. 3 Images of a tissue core containing OSCC and lymphoid tissue. (a) IHC image stained for pan-cytokeratins (dark brown), (b) FTIR image at $1285 \mathrm{~cm}^{-1}$, (c) FTIR image at $1252 \mathrm{~cm}^{-1}$ and (d) FTIR ratio image $1252 \mathrm{~cm}^{-1} / 1285 \mathrm{~cm}^{-1}$. Black arrows indicate the periphery of the tumour; white arrows identify highly keratinised areas of the tumour. Each FTIR image is plotted with a colour table covering the $5^{\text {th }}$ to $95^{\text {th }}$ percentiles of the image intensity range. Image (a) was obtained from a section adjacent to that used to obtain images (b)-(d).

ratio, a direct inspection of the region of the spectrum between $1250 \mathrm{~cm}^{-1}$ and $1289 \mathrm{~cm}^{-1}$ might be used to identify these two tissue types in similar specimens. This observation is interesting and of academic merit, but requires substantiation in a larger sample cohort than that used here. At present, it is unlikely that the current methodology would replace the standard histopathological assessment of nodal metastasis. Nonetheless, further testing of the methodology in conjunction with standard approaches in the format of a clinical trial would be of use. In addition, it would be of interest to establish whether the methodology assists in resolving the, admittedly rare, histopathological diagnostic dilemmas and challenges of detecting, for instance, isolated tumour cells or micrometastases of unusual phenotypes that are difficult to overcome via routine histology and immunohistochemistry. The present research group intends to pursue such lines of investigation in the near future. It should also be remembered that the ability to discriminate cancer from surrounding tissue on the basis of this, or any other single metric, is not necessarily true of all cancer types and it is often not possible to identify tissue types by direct inspection of spectra, as can be seen from Fig. 1 of Ingham et al. ${ }^{16}$

FTIR absorbance at $1252 \mathrm{~cm}^{-1}$ would be expected to be related to nucleic acid content. However, absorbance at this wavenumber was observed to be lower in OSCC metastasis compared with the surrounding lymphoid tissue [Fig. 3(c)] and 

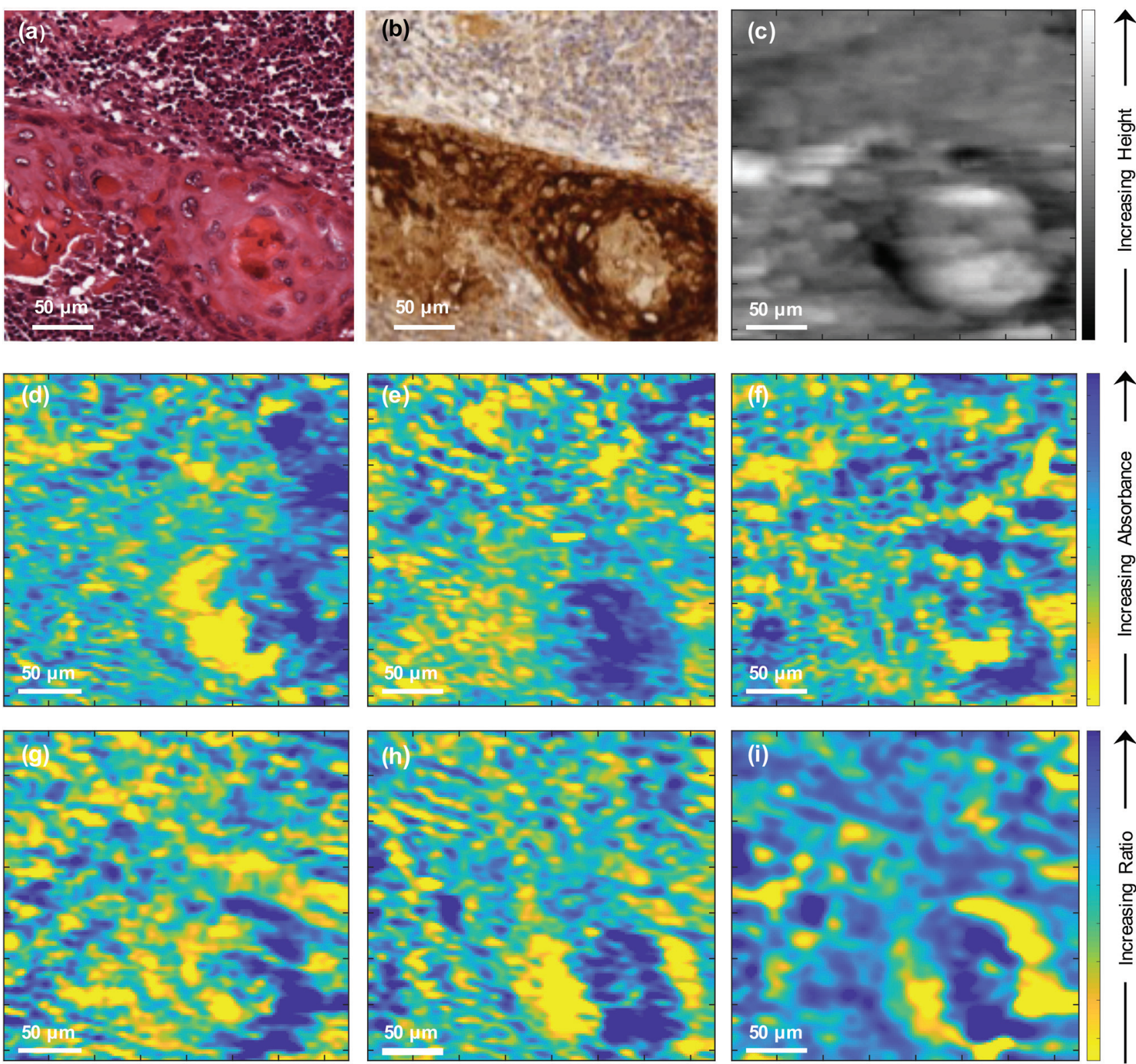

Fig. 4 (a) H\&E stained image, (b) IHC image stained for pan-cytokeratins (dark brown), (c) topography, IR SNOM images at (d) $1751 \mathrm{~cm}^{-1}$, (e) $1650 \mathrm{~cm}^{-1}$, (f) $1369 \mathrm{~cm}^{-1}$, (g) $1285 \mathrm{~cm}^{-1}$, (h) $1252 \mathrm{~cm}^{-1}$ and (i) ratio of $1252 \mathrm{~cm}^{-1} / 1285 \mathrm{~cm}^{-1}$ [i.e. (h)/(g)]. All images are $300 \mu \mathrm{m} \times 300 \mu \mathrm{m}$. Each SNOM IR image is plotted with a colour table covering the $5^{\text {th }}$ to $95^{\text {th }}$ percentiles of the image intensity range. Image (a) was obtained from a section adjacent to that used to obtain image (b), which was in turn adjacent to that used to obtain images (c) to (i).

this is reflected in the ratio of $1252 \mathrm{~cm}^{-1}$ and $1285 \mathrm{~cm}^{-1}$. This is surprising since it is known that OSSC, like many solid tumours, often shows changes in DNA ploidy ${ }^{27}$ and, indeed, that such changes may be an early event. ${ }^{28}$ This might be explained by the fact that the nuclei in lymphoid tissue are more closely packed than in the tumour, with its typically larger cells, and hence the IR absorbance at $1252 \mathrm{~cm}^{-1}$ would be higher for lymphoid tissue. The inability of FTIR to discriminate between the periphery and highly keratinised centre of the metastasis [Fig. 3(a), (c) and (d)] was overcome in higher resolution studies utilising SNOM.
The high spatial resolution of the SNOM images have the potential to provide some chemical information, although over a smaller region of the specimen and at a limited number of wavenumbers. This makes the choice of wavenumbers particularly important since biological macromolecules give complex IR absorbance spectra. Nevertheless, with a careful choice of wavenumbers, the SNOM images and line profile data, obtained with higher intrinsic spatial resolution than FTIR, can be used to infer on basic chemistry of individual tissues. The wavenumbers $1751 \mathrm{~cm}^{-1}, 1650 \mathrm{~cm}^{-1}$ and $1369 \mathrm{~cm}^{-1}$ are commonly attributed to lipids, the amide I peak of proteins 

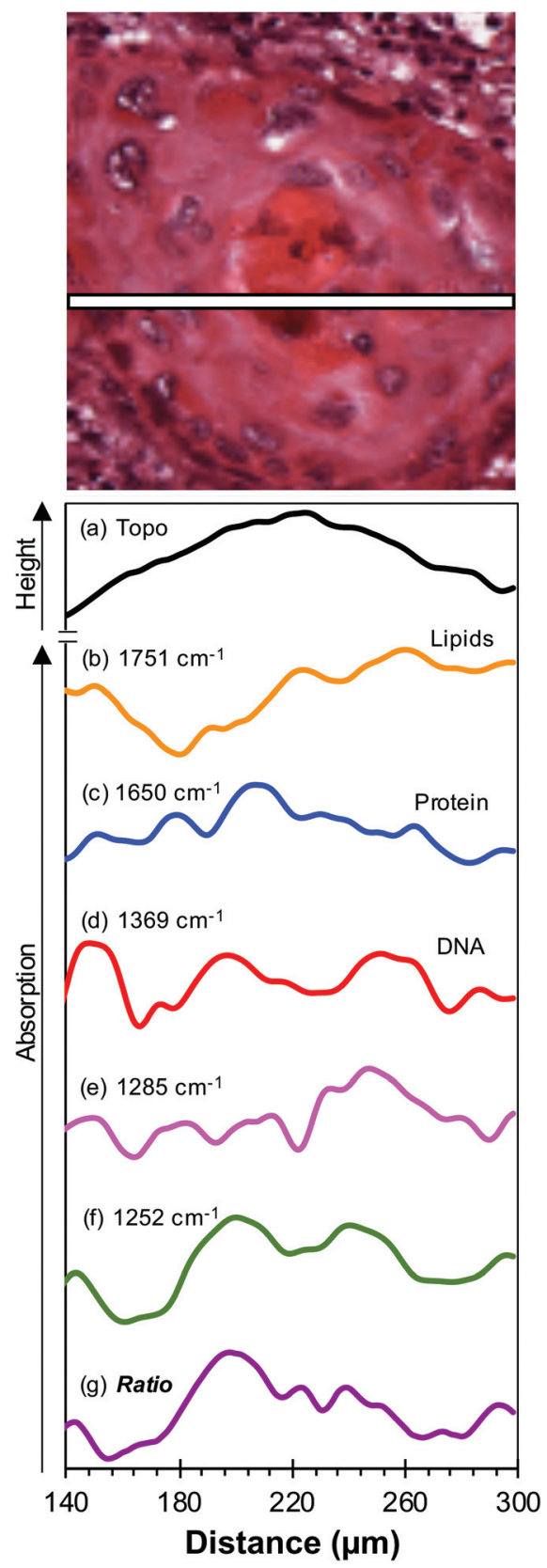

Fig. 5 H\&E stained image (top) and line profiles (bottom) taken through the core at the white line showing (a) topography, (b) $1751 \mathrm{~cm}^{-1}$, (c) $1650 \mathrm{~cm}^{-1}$, (d) $1369 \mathrm{~cm}^{-1}$, (e) $1285 \mathrm{~cm}^{-1}$, (f) $1252 \mathrm{~cm}^{-1}$ and (g) ratio of $1252 \mathrm{~cm}^{-1} / 1285 \mathrm{~cm}^{-1}$ [i.e. (f)/(e)]. H\&E image (top) was obtained from a section adjacent to that used to obtain SNOM line profiles. Each line profile has been normalised to its $\mathrm{min} / \mathrm{max}$ values.

and the $\mathrm{C}-\mathrm{N}$ stretch vibrations of the cytosine and guanine components of nucleic acids respectively ${ }^{29}$ and have been employed in previous SNOM studies, ${ }^{20,24,25}$ whereas the $1285 \mathrm{~cm}^{-1}$ signal is characteristic of collagen. ${ }^{29}$ The image obtained at $1252 \mathrm{~cm}^{-1}$ can be attributed to the $\left(\mathrm{PO}_{2}{ }^{-}\right)$nucleic acids and/or RNA signal, since this wavenumber is within a broad range of absorption from these molecules. ${ }^{30}$ As expected, the SNOM images of the small region of the tissue microarray core shown in Fig. 4 show detail on a finer length scale than is obtained in the diffraction-limited FTIR images of Fig. 3. These images show variations in the chemical structure of the tissue with a feature size down to $\sim 4 \mu \mathrm{m}$. All the images indicate differences in spectral intensities in the region of the OSCC nodal metastatic core and this region of the image is also clearly delineated in the topographic image [Fig. 4(c)]. The image formed from the ratio of the intensities of the SNOM images obtained at $1252 \mathrm{~cm}^{-1}$ and $1285 \mathrm{~cm}^{-1}$ [Fig. 4(i)] shows more contrast between different areas of the tissue than the images obtained at any of the individual wavenumbers. In particular it shows that the centre of the tumour in the bottom right of Fig. 4(a) is bounded by two broad arcs of tissue in which the ratio of the intensity of the discriminating wavenumbers is particularly low. Thus, the SNOM images are able to provide more detail than the FTIR images and highlight differences between the centre and periphery of the metastasis.

The line profiles obtained in the small region of the tumour core shown in Fig. 5 provide more detail of the chemical differences therein. As regards topography [Fig. 5(a)] the centre of the tumour was higher than the periphery. Although it is not possible to quantify this difference precisely due to the difficulty in calibrating the vertical scale of the topographic image, it was found to be $\sim 1 \mu \mathrm{m}$. The increase in height correlates with an increase in the protein signal [Fig. 5(c)] in this region of the image. The centre of the metastasis appeared highly keratinised and this is mirrored in the $1650 \mathrm{~cm}^{-1}$ amide I line profile which can be attributed to the $\alpha$-helical structure of cytokeratins. ${ }^{31,32}$ Furthermore, changes in spatial arrangement and subpopulations of cytokeratins and the molecules related to keratinisation (involucrin, etc.) are expected between the often heavily keratinised centre of tumour cells aggregates and the less keratinised periphery, the latter also corresponding to the advancing front of the primary, which could be reflected in the line profile at $1650 \mathrm{~cm}^{-1}$. In contrast to the smooth increase and decrease of both the height and the protein intensity in this region of the image, the line profiles obtained at other wavenumbers show more marked variations in intensity over smaller distances, indicating that there are subtle changes in the chemistry of the tissue. The attribution of the $1252 \mathrm{~cm}^{-1}$ signal to the $\left(\mathrm{PO}_{2}{ }^{-}\right)$ vibration of nucleic acids is supported by the very close correspondence between the line profiles obtained at $1252 \mathrm{~cm}^{-1}$ [Fig. 5(f)] and $1369 \mathrm{~cm}^{-1}$ [Fig. 5(d)], since the latter is attributed to the $\mathrm{C}-\mathrm{N}$ stretch vibrations of the cytosine and guanine components of nucleic acids. A similar correspondence between the line profiles of these two wavenumbers was found in all regions of the images examined. As previously mentioned, the line profile obtained at $1285 \mathrm{~cm}^{-1}$ [Fig. 5(e)] is attributable to collagen. However, given the relative paucity of collagen in lymph nodes, the discriminating metric of Table 1 possibly arises from variations in the levels of nucleic acids and collagen in the tissue, with the signal from the nucleic acids dominating the discrimination. This would be consistent 
with the relative discrimination between OSCC and lymphoid tissue obtained from FTIR data [Fig. 3(c) compared to Fig. 3(b)].

Taking the peak in the line profile of the topography as a reference for the centre of the tumour, the nucleic acid line profile shows a small central reduction in intensity in the centre of the metastasis with two peaks in intensity $\sim 25 \mu \mathrm{m}$ on either side, which is consistent with the increased keratinisation at that sub-site. Two further reductions in intensity are observed at $\sim 50 \mu \mathrm{m}$ from the centre and correlate with the periphery of the metastasis, with each of these features $\sim 25 \mu \mathrm{m}$ in width, roughly corresponding to 2-3 layers of cancer cells. If this signal were based solely on absorbance by nucleic acids, this would appear counter-intuitive because the more differentiated, keratinised core of the tumour most likely contains fewer, mitotically inactive nuclei compared with the tumour periphery. ${ }^{33}$ However, if we use $1252 \mathrm{~cm}^{-1}$ as a wavenumber characteristically absorbed by the phosphate groups in all nucleic acids ${ }^{34,35}$ and in the phosphate groups of phospholipids, ${ }^{36}$ we hypothesise that this increase in absorbance reflects a change in the RNA signature and/or an increase in endoplasmic reticulum commensurate with an increased proteinosynthetic events in this sub-site.

The $1285 \mathrm{~cm}^{-1}$ line profile represents a complex pattern of relative absorbance across the whole section, but notably indicates an increase immediately to the right of the tumour centre. The amount and distribution of collagen, including fibre alignment, density, width length and straightness, appear to differ between cancer types and at different sites within a tumour. ${ }^{37,38}$ These attributes have an effect on invasion, metastasis and apoptosis as well as being a prognostic factor correlated with cancer differentiation, invasion, lymph node metastasis, and clinical stage. Collagen concentration is also influenced by the hypoxic microenvironment ${ }^{39}$ and affects intensity of immune cell response. ${ }^{40}$ It is thus plausible that the differences observed in the $1285 \mathrm{~cm}^{-1}$ SNOM line profiles are due to more subtle changes in collagen fibre structure than in concentration and require further investigation.

\section{Conclusions}

A novel machine learning algorithm, MA, has been shown to accurately discriminate between OSCC nodal metastasis and surrounding lymphoid tissue on the basis of a single metric, the ratio of FTIR intensities at $1252 \mathrm{~cm}^{-1}$ and $1285 \mathrm{~cm}^{-1}$. This metric yields discriminating sensitivities, specificities and precision of $98.8 \pm 0.1 \%, 99.89 \pm 0.01 \%$ and $99.78 \pm 0.02 \%$, respectively, and an AUC of $0.9935 \pm 0.0006$. However, the topographically different periphery and highly keratinised centre of the metastasis are not discriminated by the metric in the diffraction-limited FTIR images.

SNOM images of the tissues obtained at a number of key wavenumbers, with a higher spatial resolution, show variations in chemistry with a feature size down to $\sim 4 \mu \mathrm{m}$. The image obtained from the ratio of the intensities of the SNOM images obtained at the discriminating wavenumbers supports the finding from the FTIR images that the discrimination between the two tissue types is dominated by the contribution from the $1252 \mathrm{~cm}^{-1}$ signal which is representative of nucleic acids. Additional insight into the chemistry is revealed by line profiles of the SNOM intensity obtained at specific wavenumbers, representative of particular chemical moieties, in the region of the OSCC-lymphoid tissue interface. The differences between the periphery and the centre of the metastasis reflect our current biological knowledge, but also raise additional, more subtle, questions at the cellular level.

This study demonstrates that a combination of the MA technique applied to labelled FTIR spectra together with SNOM images obtained at key wavenumbers identified by MA provides insight into the chemistry of tissues.

\section{Author contributions}

BGE designed the experiment, collected the FTIR and SNOM data, used the Metrics Analysis algorithm, prepared the figures, analysed the data, wrote MATLAB scripts used for analysis of FTIR and SNOM images and prepared the first draft. CAW wrote MATLAB scripts to analyse FTIR and SNOM data. SAJ developed the protocol for dewaxing samples for SNOM imaging. CIS designed the experiment, helped with SNOM experiments and instrumentation, prepared the figures, analysed the data, administrated the project and prepared the first draft. PJG prepared the tissue microarrays, sectioned and stained tissue and dewaxed samples for imaging. $\mathrm{PH}$ maintained the SNOM instrument and associated electronics. PU maintained the instrumentation for SNOM tip preparation. PG provided access to the FTIR Imaging microscope, supervised the FTIR experiments and mentored BGE. RJS provided the clinical methodology, supervised the work, obtained the funding and administrated the project. SDB developed the Metrics Analysis algorithm, analysed the data, supervised the work, obtained the funding administrated the project and prepared the first draft. AT designed the experiment, classified and annotated the stained samples for the supervised machine learning. JMR designed the experiment, selected the tissue samples, analysed the data, supervised the work, obtained the funding, administrated the project and prepared the first draft. PW designed the experiment, analysed the data, supervised the work, obtained the funding, administrated the project and prepared the first draft. All authors were involved in a critical review and edit of the paper.

\section{Conflicts of interest}

There are no conflicts to declare. 


\section{Acknowledgements}

The authors would like to acknowledge Cancer Research UK for funding (C7738/A26196). BGE and CAW acknowledge support from Engineering and Physical Sciences Research Council (EPSRC) PhD studentships. SAJ acknowledges the Saudi Arabia Scholarship Council for a Ph.D. studentship.

\section{References}

1 M. J. Baker, H. J. Byrne, J. Chalmers, P. Gardner, R. Goodacre, A. Henderson, S. G. Kazarian, F. L. Martin, J. Moger, N. Stone and J. Sule-Suso, Analyst, 2018, 143, 1735-1757.

2 G. R. Lloyd, L. E. Orr, J. Christie-Brown, K. McCarthy, S. Rose, M. Thomas and N. Stone, Analyst, 2013, 138, 39003908.

3 S. Berisha, M. Lotfollahi, J. Jahanipour, I. Gurcan, M. Walsh, R. Bhargava, H. Van Nguyen and D. Mayerich, Analyst, 2019, 144, 1642-1653.

4 I. P. Santos, E. M. Barroso, T. C. Bakker Schut, P. J. Caspers, C. G. F. van Lanschot, D. H. Choi, M. F. van der Kamp, R. W. H. Smits, R. van Doorn, R. M. Verdijk, V. Noordhoek Hegt, J. H. von der Thusen, C. H. M. van Deurzen, L. B. Koppert, G. van Leenders, P. C. EwingGraham, H. C. van Doorn, C. M. F. Dirven, M. B. Busstra, J. Hardillo, A. Sewnaik, I. Ten Hove, H. Mast, D. A. Monserez, C. Meeuwis, T. Nijsten, E. B. Wolvius, R. J. Baatenburg de Jong, G. J. Puppels and S. Koljenovic, Analyst, 2017, 142, 3025-3047.

5 M. Sattlecker, N. Stone and C. Bessant, Trends Anal. Chem., 2014, 59, 17-25.

6 S. Tiwari and R. Bhargava, Yale J. Biol. Med., 2015, 88, 131143.

7 M. Miljkovic, B. Bird, K. Lenau, A. I. Mazur and M. Diem, Analyst, 2013, 138, 3975-3982.

8 B. R. Smith, K. M. Ashton, A. Brodbelt, T. Dawson, M. D. Jenkinson, N. T. Hunt, D. S. Palmer and M. J. Baker, Analyst, 2016, 141, 3668-3678.

9 R. Bhargava, D. C. Fernandez, S. M. Hewitt and I. W. Levin, Biochim. Biophys. Acta, 2006, 1758, 830-845.

10 M. J. Baker, E. Gazi, M. D. Brown, J. H. Shanks, P. Gardner and N. W. Clarke, Br. J. Cancer, 2008, 99, 1859-1866.

11 M. J. Baker, E. Gazi, M. D. Brown, J. H. Shanks, N. W. Clarke and P. Gardner, J. Biophotonics, 2009, 2, 104-113.

12 T. P. Wrobel and R. Bhargava, Anal. Chem., 2018, 90, 14441463.

13 M. Pilling and P. Gardner, Chem. Soc. Rev., 2016, 45, 19351957.

14 M. Diem, A. Mazur, K. Lenau, J. Schubert, B. Bird, M. Miljkovic, C. Krafft and J. Popp, J. Biophotonics, 2013, 6, 855-886.

15 S. G. Kazarian, Spectrochim. Acta, Part A, 2021, 251, 119413.

16 J. Ingham, M. J. Pilling, D. S. Martin, C. I. Smith, B. G. Ellis, C. A. Whitley, M. R. F. Siggel-King, P. Harrison,
T. Craig, A. Varro, D. M. Pritchard, A. Varga, P. Gardner, P. Weightman and S. Barrett, Infrared Phys. Technol., 2019, 102, 103007.

17 H. J. Byrne, I. Behl, G. Calado, O. Ibrahim, M. Toner, S. Galvin, C. M. Healy, S. Flint and F. M. Lyng, Spectrochim. Acta, Part A, 2021, 252, 119470 and references therein.

18 J. D. Pallua, C. Pezzei, B. Zelger, G. Schaefer, L. K. Bittner, V. A. Huck-Pezzei, S. A. Schoenbichler, H. Hahn, A. KlossBrandstaetter, F. Kloss, G. K. Bonn and C. W. Huck, Analyst, 2012, 137, 3965-3974.

19 S. Banerjee, M. Pal, J. Chakrabarty, C. Petibois, R. R. Paul, A. Giri and J. Chatterjee, Anal. Bioanal. Chem., 2015, 407, 7935-7943.

20 S. Al Jedani, C. I. Smith, P. Gunning, B. G. Ellis, P. Gardner, S. D. Barrett, A. Triantafyllou, J. M. Risk and P. Weightman, Anal. Methods, 2020, 12, 3397-3403.

21 J. Trevisan, P. P. Angelov, A. D. Scott, P. L. Carmichael and F. L. Martin, Bioinformatics, 2013, 29, 1095-1097.

22 A. D. Smith, M. R. F. Siggel-King, G. M. Holder, A. Cricenti, M. Luce, P. Harrison, D. S. Martin, M. Surman, T. Craig, S. D. Barrett, A. Wolski, D. J. Dunning, N. R. Thompson, Y. Saveliev, D. M. Pritchard, A. Varro, S. Chattopadhyay and P. Weightman, Appl. Phys. Lett., 2013, 102, 053701.

23 C. I. Smith, M. R. F. Siggel-King, J. Ingham, P. Harrison, D. S. Martin, A. Varro, D. M. Pritchard, M. Surman, S. Barrett and P. Weightman, Analyst, 2018, 143, 59125917.

24 J. Ingham, M. J. Pilling, T. Craig, M. R. F. Siggel-King, C. I. Smith, P. Gardner, A. Varro, D. M. Pritchard, S. D. Barrett, D. S. Martin, P. Harrison, P. Unsworth, J. D. Kumar, A. Wolski, A. Cricenti, M. Luce, M. Surman, Y. M. Saveliev and P. Weightman, Biomed. Phys. Eng. Express, 2018, 4, 025011.

25 J. Ingham, T. Craig, C. I. Smith, A. Varro, D. M. Pritchard, S. D. Barrett, D. S. Martin, P. Harrison, P. Unsworth, J. D. Kumar, A. Wolski, A. Cricenti, M. Luce, M. Surman, Y. M. Saveliev, P. Weightman and M. R. F. Siggel-King, Biomed. Phys. Eng. Express, 2019, 5, 015009-015019.

26 M. A. Unger, D. A. Kossakovski, R. Kongovi, J. L. Beauchamp and J. D. Baldeschwieler, Rev. Sci. Instrum., 1998, 69, 2988-2993.

27 P. R. Bueno, L. N. Gias, R. G. Delgado, J. D. Cebollada and F. D. Gonzalez, Head Neck, 1998, 20, 232-239.

28 H. P. Sathasivam, D. Nayar, P. Sloan, P. J. Thomson, E. W. Odell and M. Robinson, J. Oral Pathol. Med., 2021, 50, 200-209.

29 A. C. S. Talari, M. A. G. Martinez, Z. Movasaghi, S. Rehman and I. U. Rehman, Appl. Spectrosc. Rev., 2017, 52, 456-506.

30 P. Zucchiatti, E. Mitri, S. Kenig, F. Bille, G. Kourousias, D. E. Bedolla and L. Vaccari, Anal. Chem., 2016, 88, 1209012098.

31 P. M. Steinert, Biochem. J., 1975, 149, 39-48.

32 H. H. Bragulla and D. G. Homberger, J. Anat., 2009, 214, 516-559.

33 M. Bryne, Oral Dis., 1998, 4, 70-77. 
34 H. P. Wang, H. C. Wang and Y. J. Huang, Sci. Total Environ., 1997, 204, 283-287.

35 B. Rigas, S. Morgello, I. S. Goldman and P. T. Wong, Proc. Natl. Acad. Sci. U. S. A., 1990, 87, 8140-8144.

36 H. Fabian, M. Jackson, L. Murphy, P. H. Watson, I. Fichtner and H. H. Mantsch, Biospectroscopy, 1995, 1, 37-45.

37 S. Xu, H. Xu, W. Wang, S. Li, H. Li, T. Li, W. Zhang, X. Yu and L. Liu, J. Transl. Med., 2019, 17, 309.
38 B. F. Matte, A. Kumar, J. K. Placone, V. G. Zanella, M. D. Martins, A. J. Engler and M. L. Lamers, J. Cell Sci., 2019, 132, jcs224360.

39 S. M. Kakkad, M. Solaiyappan, B. O'Rourke, I. Stasinopoulos, E. Ackerstaff, V. Raman, Z. M. Bhujwalla and K. Glunde, Neoplasia, 2010, 12, 608-617.

40 H. Bougherara, A. Mansuet-Lupo, M. Alifano, C. Ngo, D. Damotte, M. A. Le Frere-Belda, E. Donnadieu and E. Peranzoni, Front. Immunol., 2015, 6, 500. 